K-ras is currently accepted as the most frequently mutated oncogene in nonsmall cell lung cancer (NSCLC, including squamous carcinoma, adenocarcino$\mathrm{ma}$, and large cell carcinoma). NSCLC patients with the K-ras mutation appear to be refractory to the majority of systemic therapies. In the present study, the in vitro antitumor effects and correlated molecular mechanisms of sorafenib combined with gemcitabine or pemetrexed were explored in the K-ras mutation-positive NSCLC A549 cell line. Sorafenib was seen to exhibit dose-dependent growth inhibition in the A549 cells, while sorafenib combined with pemetrexed demonstrated a greater synergism compared with sorafenib combined with gemcitabine. Sorafenib arrested the cell cycle at the G1 phase, while gemcitabine and pemetrexed caused arrest at the $\mathrm{S}$ phase. The molecular mechanism of this synergism was due to the downstream signalling pathways, which were efficiently suppressed by sorafenib, therefore increasing the incidence of the entry of the chemotherapeutic drugs into the apoptotic pathways. Moreover, sorafenib and pemetrexed demonstrated stronger synergism, demonstrating that inhibiting the Ras/Raf/Mek/Erk and Ras/ PI3K/Akt pathways concurrently may achieve improved antitumor effects.

Key words: sorafenib, gemcitabine, pemetrexed, lung cancer, K-ras mutations, western blot.

Contemp Oncol (Pozn) 2016; 20 (1): 33-38 DOI: $10.5114 /$ wo.2016.58499

\section{Synergistic effects of sorafenib in combination with gemcitabine or pemetrexed in lung cancer cell lines with K-ras mutations}

\author{
Shu Wang ${ }^{1}$, Zeng-feng Su${ }^{1}$, Yuan Yuan ${ }^{2}$, Jing $\mathrm{Li}^{2}$ \\ ${ }^{1}$ Hefei Binhu Hospital, China \\ ${ }^{2}$ Anhui Provincial Hospital Affiliated to Anhui Medical University, China
}

\section{Introduction}

Recent data has shown that chemotherapy for non-small cell lung cancer (NSCLC) has reached a therapeutic plateau for overall survival and quality of life [1, 2]. At the same time, the small-molecule epidermal growth factor receptor (EGFR) tyrosine kinase inhibitors (TKIs) that improve the survival of NSCLC patients are causing a paradigm shift in the treatment of NSCLC. Patients with EGFR-activating mutations may greatly benefit from treatment with EGFR TKIs [3-5]. However, K-ras (codon 2 and exon 12) mutations have been correlated with non-responsiveness to EGFR TKIs [6, 7] and systemic chemotherapy [8]. K-ras mutations are found with a high frequency in a variety of human tumours, including $20-40 \%$ of NSCLC cases [9]. Thus, clinical research of new treatment strategies for NSCLC patients with K-ras mutations is urgently required.

The Ras/Raf/Mek/Erk and Ras/PI3K/Akt pathways interact with each other to regulate growth, and they play key roles in the transmission of proliferative signals in tumorigenesis. In NSCLC, the activation of K-ras leads to ERK1/2 overexpression through the Raf-Mek-Erk signalling pathway [10-12]. Sorafenib (BAY 43-9006) is an oral multikinase inhibitor that decreases the activity of C-raf and B-raf in the Raf/Mek/Erk signalling pathway and targets the vascular endothelial growth factor receptor (VEGFR-2 and VEGFR-3), platelet-derived growth factor receptor- $\beta$ (PDGFR- $\beta$ ) families, fibroblast growth factor receptor (FGFR-1), as well as FLT-3, C-KIT, and RET [13]. Sorafenib has been shown to inhibit the growth of a variety of tumour xenograft models driven by the upregulation of Raf/MEK/ERK signalling [14]. Single-agent sorafenib has shown clinical activity in heavily pretreated patients with NSCLC. Stable disease was observed in 30 of 51 (59\%) patients treated with 400 mg sorafenib twice daily [15]. In xenograft models, when combining sorafenib with anti-cancer agents, including vinorelbine, cisplatin, and gefitinib, the anti-proliferative effect is at least as effective as sorafenib alone and is well-tolerated [16]. The safety profile of sorafenib in previous trials has increased the feasibility of its use in combination with cytotoxic and cytostatic agents.

Gemcitabine is a pyrimidine nucleoside antimetabolite agent with a favourable toxicity profile, which is active against a variety of human malignancies, particularly NSCLC [17]. Gemcitabine has frequently been used in combinatorial treatments with other anticancer agents.

Pemetrexed is a new antifolate that has significant activity against a broad spectrum of solid tumours, including lung cancer [18, 19]. Pemetrexed inhibits multiple enzymes involved in folate metabolism, including thymidylate synthase, dihydrofolate reductase, and glycinamide ribonucleotide formyltransferase, thereby depleting nucleotide pools and blocking DNA synthesis [20, 21]. 
In the present study, in vitro NSCLC cells harbouring K-ras mutations were used to investigate the effect of sorafenib combined with gemcitabine or pemetrexed on proliferation and cell cycle progression and the molecular mechanisms behind the synergistic effects.

\section{Material and methods}

\section{Drugs}

Sorafenib (BAY 43-9006) was obtained from Bayer (Leverkusen, Germany) and dissolved in dimethyl sulfoxide (DMSO) to a stock concentration of $10 \mathrm{mmol} / \mathrm{l}$. Gemcitabine and pemetrexed were gifts provided by Eli Lilly that were then dissolved in DMSO at $100 \mathrm{mmol} / \mathrm{l}$ as the stock solution. The drugs were stored at $-20^{\circ} \mathrm{C}$ and diluted with culture medium prior to use.

\section{Cell lines}

The K-ras mutation human NSCLC A549 cell line was purchased from the American Type Culture Collection (ATCC, Manassas, VA, USA) and maintained in RPMI-1640 medium (Hyclone, Waltham, MA, USA), supplemented with 10\% heat-inactivated foetal bovine serum (Hyclone), penicillin $(100 \mathrm{U} / \mathrm{ml})$, streptomycin $(100 \mathrm{\mu g} / \mathrm{ml})$, and L-glutamine $(2 \mathrm{mM})$ at $37^{\circ} \mathrm{C}$ in $5 \% \mathrm{CO}_{2}$. The cells were then harvested with trypsin-EDTA when they had reached a point of exponential growth.

\section{Anti-proliferative effects of single agents}

The anti-proliferative effects of sorafenib, gemcitabine, and pemetrexed as single agents on the A549 cells were evaluated using an MTT assay, as previously described [22]. The cells (4000 cells per well) were cultured in 96-well plates. The IC50 value, indicating the concentration resulting in inhibition of $50 \%$ of the maximal cell growth, was determined following 72 hours of exposure to the drugs, as compared with unexposed control cells. Subsequent to the cells being exposed to each drug for 72 hours in 96 well plates, $20 \mu \mathrm{l}$ MTT solution was added to each well. The optical density (OD) of each well was measured at $490 \mathrm{~nm}$ following incubation for 4 hours. The percentage of cell growth inhibition resulting from each drug was calculated as: (OD of 490 control cells - OD of 490 treated cells) / OD of 490 control cells $\times 100$. This assay was repeated in more than three independent experiments.

\section{Anti-proliferative effects of sorafenib combined with gemcitabine or pemetrexed}

A549 cells were concurrently exposed to sorafenib combined with gemcitabine or pemetrexed for 72 hours. The combination drug doses were selected using constant ratios of the IC50 values. Thus, the combination index $(\mathrm{Cl})$ value was calculated using $0.125,0.25,0.5,1,2$, and 4 times the IC50 of the drug combination doses. The $\mathrm{Cl}$ values were analysed according to the method used by Chou and Talaly using CompuSyn software (ComboSyn, Inc., Paramus, NJ, USA). $\mathrm{Cl}>1, \mathrm{Cl}=1$, and $\mathrm{Cl}<1$ indicated antagonistic, additive, and synergistic effects, respectively [23].

\section{Cell cycle analysis}

The cells $\left(1 \times 10^{5} /\right.$ well $)$ were plated onto 6 -well plates and exposed to sorafenib and gemcitabine (or pemetrexed) as single agents and in combination at the concentration of the IC50 levels for 72 hours. At the end of each exposure, the cells were collected and fixed with $70 \%$ cold ethanol at $4^{\circ} \mathrm{C}$ overnight. DNA staining was performed using a solution with propidium iodide $(0.05 \mathrm{mg} / \mathrm{ml})$ and RNase $(2 \mathrm{mg} / \mathrm{ml})$ for 30 minutes at room temperature. The cells were analysed using a FACScan cytometer (Becton-Dicknson, Franklin Lakes, NJ, USA), and the percentage of cells in G1, S, and G2 phases of the cell cycle was estimated using Cell Lab Quanta SC software (Beckman Coulter, Miami, FL, USA).

\section{Western blot analysis}

The cells $\left(5 \times 10^{5} /\right.$ well $)$ were treated with sorafenib and gemcitabine (or pemetrexed) as single agents and in combination at the concentration of the IC50 levels for 72 hours. The cells were washed with ice-cold phosphate-buffered saline (PBS) solution and scraped in lysis buffer. The lysates were centrifuged at 14,000 rpm for 30 minutes at $4^{\circ} \mathrm{C}$ and the supernatant was collected. Equivalent amounts of protein were analysed by sodium dodecyl sulphate-polyacrylamide gel electrophoresis (SDS-PAGE) and then transferred to PVDF membranes. Appropriate primary antibodies to PAKT, AKT, pERK1/2, ERK1/2, Bcl-2, and $\beta$-actin, purchased from Cell Signaling Technology (Beverly, MA, USA), were used. The proteins were visualised with a horseradish peroxidase-coupled secondary antibody from Cell Signaling Technology. Specific bands were detected using an enhanced chemiluminescence reagent (ECL; Perkin-Elmer Life Sciences, Inc., Boston, MA, USA) on autoradiographic film and quantitated by densitometry.

\section{Statistical analysis}

The results obtained from at least three independent experiments are expressed as the mean \pm standard deviation. The Student's $t$ test and one-way ANOVA test were used to determine the differences between the control and treatment groups. $P<0.05$ was considered to indicate a statistically significant difference.

\section{Results}

\section{Anti-proliferative effects}

Sorafenib, gemcitabine, and pemetrexed demonstrated a dose- and time-dependent anti-tumoural effect in the A549 cells when used as single agents (Fig. 1). Subsequent to a 72-hour exposure, the IC50 values of sorafenib, gemcitabine, and pemetrexed in the A549 cells were 4.09 $\pm 0.16,11.54 \pm 0.77$, and $3.90 \pm 0.40 \mu \mathrm{M}$, respectively.

When exposed to the concurrent administration of sorafenib and gemcitabine, synergistic activity (mean Cl value, 0.86) was observed in the A549 cells. Moreover, the concurrent administration of sorafenib and pemetrexed generated a greater synergistic effect (mean CI value, 0.63; Figs. 2 and 3). 


\section{Cell cycle effects}

Flow cytometry was applied to evaluate the cell cycle phase distributions in the A549 cells following single-drug and concurrent administration of sorafenib and gemcitabine (or pemetrexed) for 72 hours. Following sorafenib treatment, the proportion of G0/G1 phase cells increased relative to the control values $(p<0.01)$. With gemcitabine or pemetrexed treatment alone, the fraction of $S$ phase cells increased $(p<0.01)$.

Upon exposure to a concurrent administration of sorafenib and gemcitabine, the proportion of G0/G1 phase and S-phase cells increased compared with the control $(p<0.05)$. However, when exposed to sorafenib combined with pemetrexed, the proportion of GO/G1 phase cells decreased, while the proportion of $\mathrm{G} 2 / \mathrm{M}$ phase cells markedly increased (Fig. 4).

\section{Effects on downstream signalling pathways}

Following a 72-hour exposure at the IC50 concentration, gemcitabine was demonstrated to upregulate the levels of $p-E R K, p-A K T$, and $\mathrm{BCl}-2$, whereas pemetrexed upregulated the levels of $\mathrm{p}$-ERK and $\mathrm{BCl}-2$, but decreased the level of $\mathrm{p}-\mathrm{AKT}$, and sorafenib downregulated the levels of $p$-ERK, p-AKT, and Bcl-2, compared with the control A549 cells.

In addition, when exposed to a concurrent administration of sorafenib and gemcitabine, the levels of $p$-ERK and $\mathrm{BCl}-2$ were downregulated, while the level of $\mathrm{p}$-AKT remained identical to that of the control group. When exposed to sorafenib combined with pemetrexed for 72 hours, the levels of $\mathrm{p}$-ERK and $\mathrm{BCl}-2$ decreased and the level of p-AKT further declined (Fig. 5).

\section{Discussion}

K-ras is currently accepted to be the most frequently mutated oncogene in NSCLC. It represents the activating mutation in the Ras/Raf/Mek/Erk signalling pathway. Sorafenib is a novel, multikinase inhibitor that targets tumour proliferation and angiogenesis [13]. The present study was performed on the K-ras mutation A549 human lung cancer cell line to investigate the anti-proliferative effects of sorafenib as a single agent and in combination with chemotherapy using gemcitabine or pemetrexed.

Sorafenib, gemcitabine, and pemetrexed were demonstrated to exhibit dose-dependent growth inhibition of cell growth in the A549 cells when used as single agents. The combination of sorafenib with gemcitabine or pemetrexed generated synergistic effects in the A549 cells. Moreover, greater synergism was observed upon use of sorafenib combined with pemetrexed.

In the present study, the A549 cells were arrested at the G1 phase by sorafenib, while gemcitabine and pemetrexed caused S-phase cell accumulation. When exposed to a concurrent administration of sorafenib and gemcitabine, the proportion of G0/G1 phase and S-phase cells increased. These effects on the cell cycle may explain the synergism observed between the two drugs, since the two pharmacological agents did not antagonise their mutual mechanism of action.
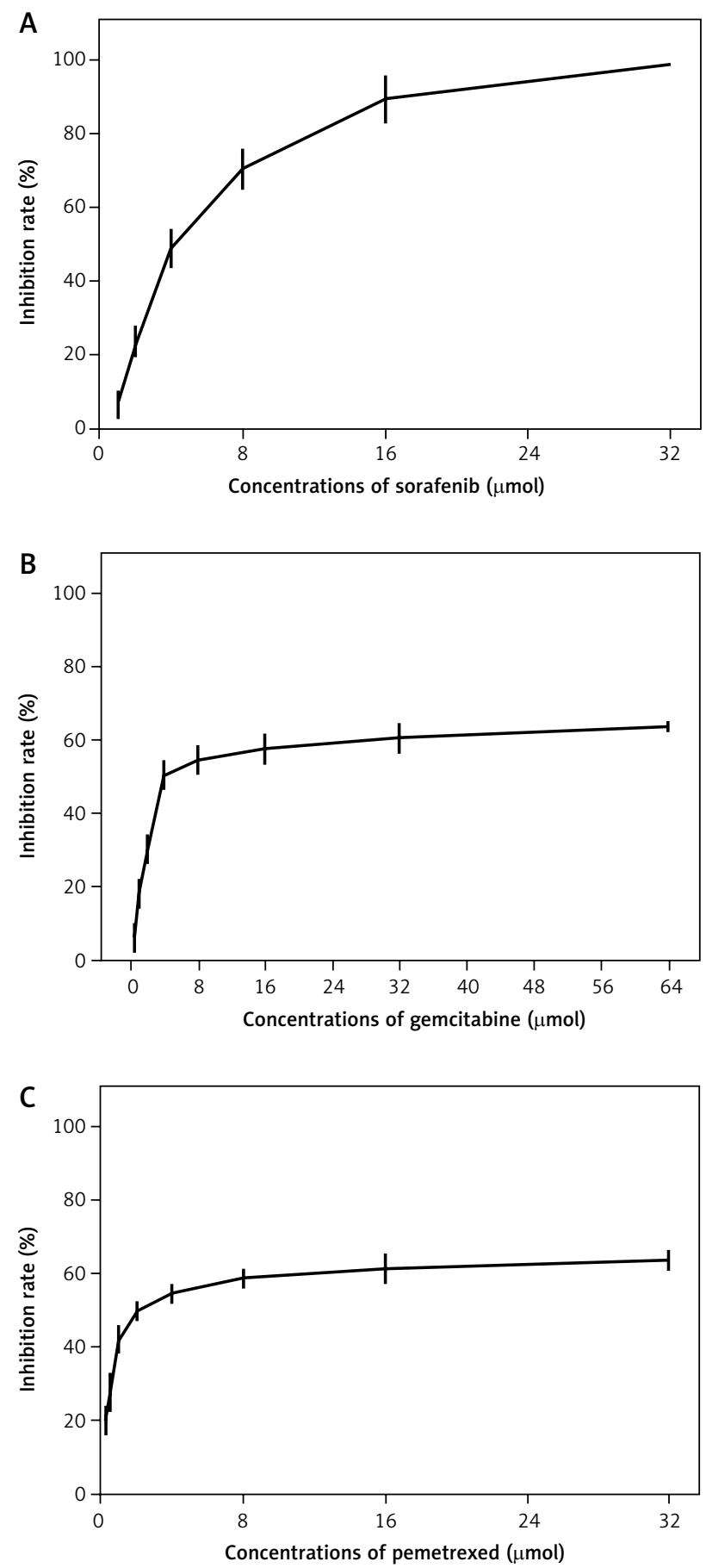

Fig. 1. In vitro effects of sorafenib, gemcitabine, and pemetrexed on proliferation of A549 cell lines. MTT assays were used to examine the inhibitory activities on cell proliferation. Each data point was repeated in more than three independent experiments

When exposed to sorafenib combined with pemetrexed, the proportion of G0/G1 phase cells was significantly reduced, while the proportion of G2/M phase cells was significantly increased. This may be due to the fact that the cells in the G1 phase entered into the apoptotic pathway and died, so the proportion in the G2/M phase was relatively increased.

The present study also found that gemcitabine enhanced the expression levels of certain downstream sig- 



Fig. 2. A549 cells were exposed to different combinations at IC50 levels. (OLYMPUS IX70 ×200 magnification). The anti-proliferative effects of sorafenib combined with pemetrexed were more pronounced than sorafenib combined with gemcitabine

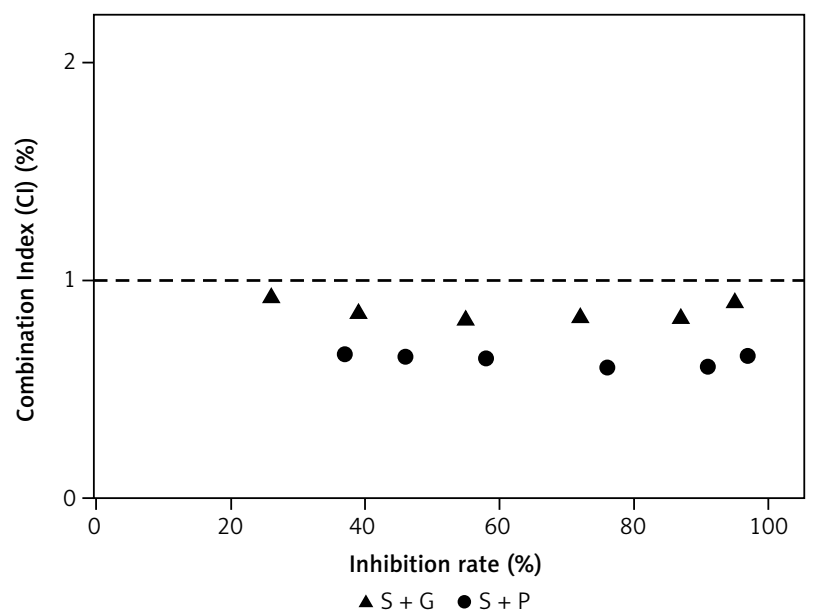

Fig. 3. The combination index $(\mathrm{Cl})$ value of the two combinations was calculated using the Chou-Talalay method. S+G means sorafenib and gemcitabine, S+P means sorafenib and pemetrexed. $\mathrm{Cl}<1$ was detected in both of the two combinations; sorafenib and pemetrexed generated stronger synergistic effects

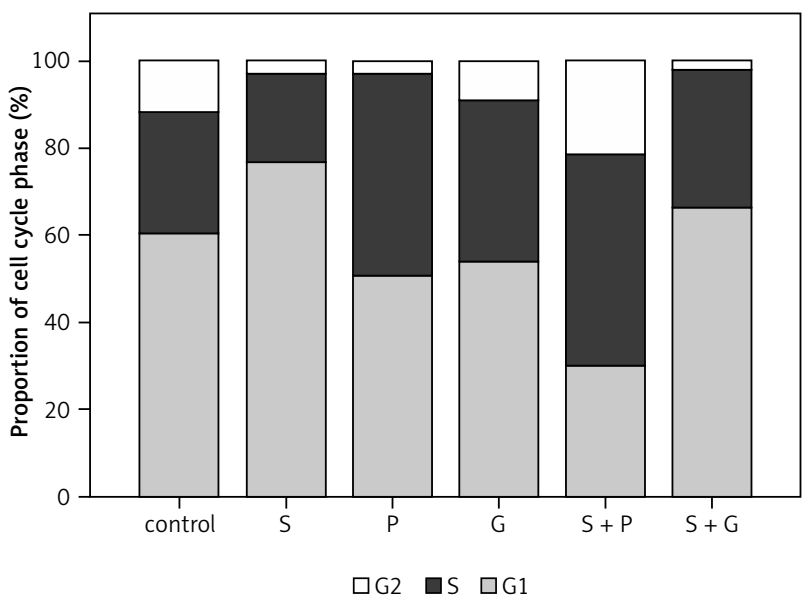

Fig. 4. Flow cytometric analysis was applied to determine the alterations in cell cycle distributions in A549 cell lines. The concentrations of sorafenib, gemcitabine, and pemetrexed were used at IC50 levels. Columns in the diagram depict cell cycle phase distribution in A549 cells 

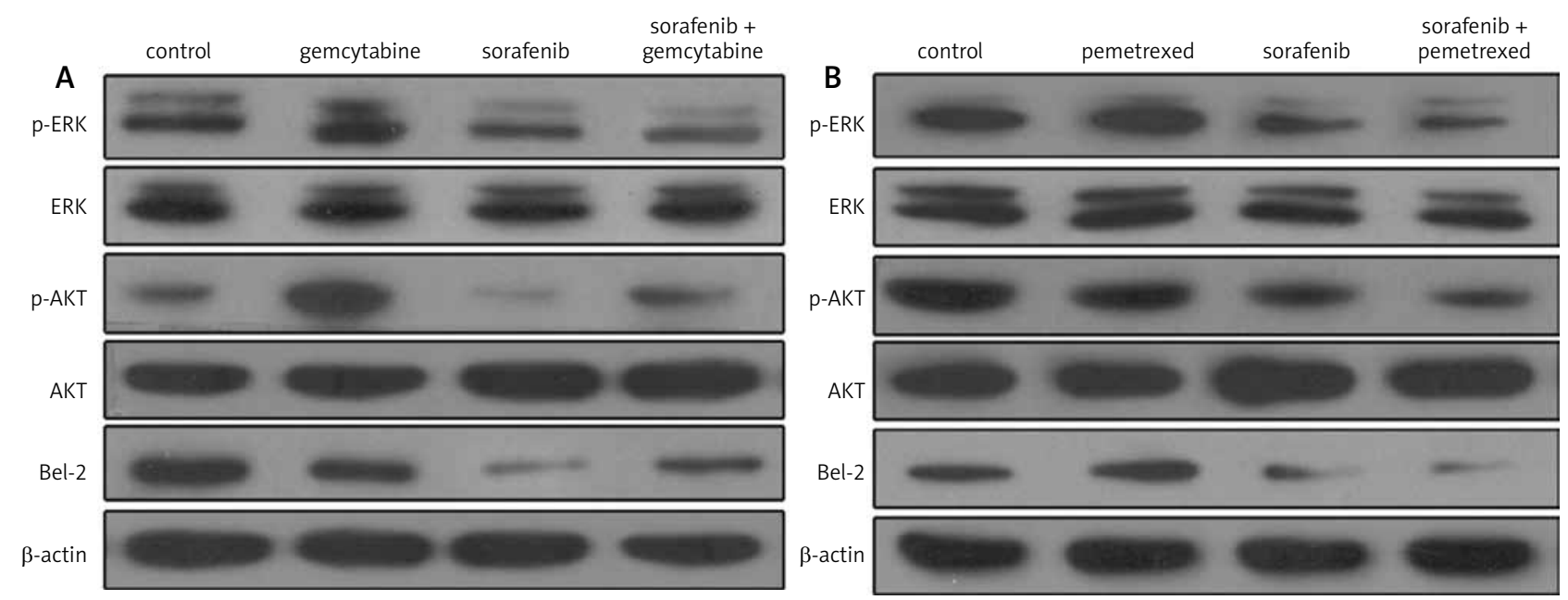

Fig. 5. The expression levels of downstream signalling pathways in A549 cells were evaluated by western blot analysis

nalling pathways, increasing the levels of p-AKT, p-ERK and $\mathrm{BCl}-2$ in the $\mathrm{A} 549$ cells. Pemetrexed also enhanced the expression levels of $\mathrm{p}$-ERK and Bcl-2. In accordance with these results, a previous study reported that the cell signalling pathways may be gradually activated by chemotherapy [24]. p-ERK and p-AKT play significant roles in tumour cell proliferation; chemotherapy increases the levels of $p$-ERK and p-AKT, leading to the prevention of apoptosis. We also found that sorafenib may downregulate the levels of downstream p-AKT, p-ERK, and Bcl-2 in the A549 cell lines following 72 hours of exposure.

When sorafenib was combined with gemcitabine or pemetrexed, a significant decrease was observed in the p-ERK and Bcl-2 levels in the A549 cells compared with the control cells. We conclude that the RAF/MEK/ERK signalling pathways activated by gemcitabine or pemetrexed may be blocked by sorafenib, thus increasing the incidence of the entry of the chemotherapeutic drugs into the apoptotic pathways. These observations on $\mathrm{p}$-ERK and Bcl-2 in NSCLC cells may explain the synergistic growth inhibitory effects of sorafenib combined with gemcitabine or pemetrexed.

In the present study, pemetrexed and sorafenib downregulated the level of $\mathrm{p}$-AKT individually, and when the two drugs were combined the level of p-AKT was further decreased. When using a combination of sorafenib and gemcitabine, the level of p-AKT was neither increased by the gemcitabine nor decreased by the sorafenib, but remained identical to that of the control group. Thus, this is why the combination of sorafenib and pemetrexed generates a stronger synergistic effect than sorafenib and gemcitabine. The simultaneous inhibition of $p$-AKT and $p$-ERK generates greater anti-proliferative effects.

In conclusion, we found that sorafenib caused significant growth inhibition in the NSCLC cells with K-ras mutation. Moreover, sorafenib combined with pemetrexed generated greater synergistic effects than sorafenib and gemcitabine against the NSCLC cells with K-ras mutation. These data are encouraging for the development of sorafenib as a single targeted therapy or in combination with cytotoxic chemotherapy drugs for the treatment of NSCLC cells with K-ras mutation. Further studies are need- ed to explore in vivo sorafenib as a single agent and combined with gemcitabine or pemetrexed in lung cancer cell lines with K-ras mutations.

The authors declare no conflict of interest.

\section{References}

1. Scagliotti GV, Parikh P, von Pawel J, et al. Phase III study comparing cisplatin plus gemcitabine with cisplatin plus pemetrexed in chemotherapy-naive patients with advanced-stage non-small-cell lung cancer. J Clin Oncol 2008; 26: 3543-51.

2. Eldeeb H, Reza S. Northampton outcome for first and second line chemotherapy in non-small cell lung cancer: 5 years data. Contemp Oncol (Pozn) 2012; 16: 420-3.

3. Mok TS, Wu YL, Thongprasert S, et al. Gefitinib or carboplatin-paclitaxel in pulmonary adenocarcinoma. N Engl J Med 2009; 361: 947-57.

4. Kowalski DM, Krzakowski M, Ramlau R. Erlotinib in salvage treatment of patients with advanced non-small cell lung cancer: results of an expanded access programme in Poland. Contemp Oncol (Pozn) 2012; 16: 170-5.

5. Płużański A, Piórek A, Krzakowski M. Crizotinib in the treatment of non-small-cell lung cancer. Contemp Oncol (Pozn) 2012, 16: 480-4.

6. Pao W, Wang TY, Riely GJ, et al. KRAS mutations and primary resistance of lung adenocarcinomas to gefitinib or erlotinib. PLoS Med 2005; 2: e17.

7. Gazdar AF. Activating and resistance mutations of EGFR in nonsmall-cell lung cancer: role in clinical response to EGFR tyrosine kinase inhibitors. Oncogene 2009; 28 (Suppl 1): S24-S31.

8. Rodenhuis S, Slebos RJ, Boot AJ, et al. Incidence and possible clinical significance of K-ras oncogene activation in adenocarcinoma of the human lung. Cancer Res 1988; 48: 5738-41.

9. Lewandowska MA, Jóźwicki W, Starzyński J, et al. Analysis of EGFR mutation frequency and coexistence of KRAS and EGFR mutations using RT-PCR in lung adenocarcinoma: may a clinical and pathological model of a patient's qualification for targeted therapy have an impact on time to obtain genetic results? Kardiochir Torakochir Pol 2012; 9: 443-51.

10. Linardou H, Dahabreh IJ, Kanaloupiti D, et al. Assessment of somatic k-Ras mutations as a mechanism associated with resistance to EGFR-targeted agents: a systematic review and meta-analysis of studies in advanced non-small-cell lung cancer and metastatic colorectal cancer. Lancet Oncol 2008; 9: 962-72.

11. Rodenhuis S, van de Wetering ML, Mooi WJ, et al: Mutational activation of the K-ras oncogene. A possible pathogenetic factor in adenocarcinoma of the lung. N Engl J Med 1987; 317: 929-35. 
12. Lopez-Chavez A, Carter CA, Giaccone G. The role of KRAS mutations in resistance to EGFR inhibition in the treatment of cancer. Curr Opin Investig Drugs 2009; 10: 1305-14.

13. Wilhelm SM, Carter C, Tang L, et al. BAY 43-9006 exhibits broad spectrum oral antitumor activity and targets the RAF/MEK/ERK pathway and receptor tyrosine kinases involved in tumor progression and angiogenesis. Cancer Res 2004; 64: 7099-109.

14. Suzuki Y, Orita M, Shiraishi M, Hayashi K, Sekiya T. Detection of ras gene mutations in human lung cancers by single-strand conformation polymorphism analysis of polymerase chain reaction products. Oncogene 1990; 5: 1037-43.

15. Blumenschein GR, Gatzemeier U, Fossella F, et al. Phase II, multicenter, uncontrolled trial of single-agent sorafenib in patients with relapsed or refractory, advanced non-small-cell lung cancer. J Clin Oncol 2009; 27: 4274-80.

16. Carter CA, Chen C, Brink C, et al. Sorafenib is efficacious and tolerated in combination with cytotoxic or cytostatic agents in preclinical models of human non-small cell lung carcinoma. Cancer Chemother Pharmacol 2007; 59: 183-95.

17. Dougherty DW, Friedberg JW. Gemcitabine and other new cytotoxic drugs: will any find their way into primary therapy? Curr Hematol Malig Rep 2010; 5: 148-56.

18. Adjei AA. Pemetrexed (ALIMTA), a novel multitargeted anti-neoplastic agent. Clin Cancer Res 2004; 10 suppl: 4276S-80S

19. Hanna N, Shepherd FA, Fossella FV, et al. Randomized phase III trial of pemetrexed versus docetaxel in patients with non-smallcell lung cancer previously treated with chemotherapy. J Clin Oncol 2004; 22: 1589-97.

20. Shih C, Chen VJ, Gossett LS, et al. LY231514, a pyrrolo(2, 3-d)pyrimidine-based antifolate that inhibits multiple folate-requiring enzymes. Cancer Res 1997; 57: 1116-23.

21. Pandita TK, Lieberman HB, Lim DS, et al. Ionizing radiation activates the ATM kinase throughout the cell cycle. Oncogene 2000; 19: 1386-91.

22. Mosmann T. Rapid colorimetric assay for cellular growth and survival: application to proliferation and cytotoxicity assays. J Immu nol Methods 1983; 65: 55-63.

23. Chou TC, Talalay P. Quantitative analysis of dose-effect relationships: the combined effects of multiple drugs or enzyme inhibitors. Adv Enzyme Regul 1984; 22: 27-55.

24. Torres K, Horwitz SB. Mechanisms of Taxol-induced cell death are concentration dependent. Cancer Res 1998; 58: 3620-6.

\section{Address for correspondence}

Jing Li

Anhui Provincial Hospital Affiliated to Anhui Medical University

17 Lujiang Road

233000 Hefei, China

e-mail: 15055160501@163.com

Submitted: 28.10 .2013

Accepted: 11.06 .2014 